Article

\title{
The Mixed-Electrode Concept for Understanding Growth and Aggregation Behavior of Metal Nanoparticles in Colloidal Solution
}

\author{
Johann Michael Köhler* (D) and Andrea Knauer \\ Institute for Micro- und Nanotechnologies/Institute for Chemistry and Biotechnology, Department for Physical \\ Chemistry and Microreaction Technology, Ilmenau Technology University, D-98693 Thüringen, Germany; \\ andrea.knauer@tu-ilmenau.de \\ * Correspondence: michael.koehler@tu-ilmenau.de
}

Received: 29 June 2018; Accepted: 3 August 2018; Published: 10 August 2018

Featured Application: Insight into the electrochemical nature of the growth of metal nanoparticles supports the development of new concepts and protocols for the production of non-spherical nanoparticles of high homogeneity, for control of particle geometry and for the development of new types of nanoparticles.

\begin{abstract}
The growth and aggregation behavior of metal nanoparticles can be modulated by surfactants and different other additives. Here the concept of how open-circuit mixed electrodes helps to understand the electrical aspects of nanoparticle growth and the consequences for the particle geometries is discussed. A key issue is the self-polarization effect of non-spherical metal nanoparticles, which causes a local decoupling of anodic and partial processes and asymmetry in the local rates of metal deposition. These asymmetries can contribute to deciding to the growth of particles with high aspect ratios. The interpretation of electrochemical reasons for particle growth and behavior is supported by experimental results of nanoparticle syntheses supported by microfluidics which can supply high yields of non-spherical nanoparticles and colloidal product solutions of high homogeneity.
\end{abstract}

Keywords: metal nanoparticles; particle growth; anisotropy; mixed electrode; self-polarization; aspect ratios

\section{Introduction}

Metal nanoparticles and their colloidal solutions have attracted a lot of attention in research in recent decades. Despite many publications on synthesis, formation mechanisms [1] and application of these nanoparticles [2], there exists a lack of understanding in some aspects of the growth and behavior of nanoparticles of special shapes and compositions. One rather successful approach comes from the direction of molecular modelling and can describe the formation of clusters of a lower and well-defined number of atoms [3]. Typically, such an approach is based on the optimization of thermodynamic conditions. These models are based on the identification of states and structures with minimum energy. This approach has the character of a bottom-up description.

An alternative approach follows a top-down strategy and regards the metal nanoparticles from the point of view of small solids. It is marked mainly by the down-scaling of properties, parameters, structures and behavior from larger metal solids to the nanoscale. It is obvious that such an approach must pay a lot of attention to the crystallographic structure, lattice parameters and surfaces $[4,5]$. One consequence of this point of view is the trial to explain many of the observations during the 
growth and reaction of nanoparticles by differences in the properties of crystallographic planes at the surface of nanocrystals and by differences in the interaction of these surface planes with ligands in solution $[6,7]$.

The experimental proof of an initial nucleation/aggregation mechanism for spherical gold nanoparticles was a milestone in the understanding of the formation of colloidal metal nanoparticles [8]. In situ X-ray investigations in a flow synthesis process allowed monitoring of the formation of small nuclei, their fast aggregation to medium-sized nanoparticles and the subsequent self-stabilization of the colloidal solution on a certain size level of metal nanoparticles. The detection of this mechanism explained the formation of nearly spherical metal particles, their comparatively high size homogeneity, and their polynuclear character [9]. The principle of combined spontaneous aggregation of colloidal particles followed by self-stabilization corresponds to the aggregation/re-stabilization mechanism which was also observed on larger star-shaped gold/silver nanoparticles [10].

Despite proving the above-mentioned nucleation/aggregation mechanism, other aspects of nanoparticle formation remained unexplained, are an object of mechanistic speculations, and are regarded as a kind of mystery [11]. It is astonishing that such low attention has been paid to the role of electrical properties of metal nanoparticles for the formation of particle geometries, although the majority of researchers are aware that colloidal particles are carrying charges in most cases, and charge strongly influences the transport and aggregation behavior [12]. In particular, the discussions on the reasons of formation of non-spherical nanoparticles is frequently discussed under the aspects of crystal growth and connected to speculations on the selective covering of certain surface parts by ligands [13-15] without any respect to the electrical properties of nanoparticles. Important insights into the role of electrostatic and electrochemical effects on the growth and behavior of nanoparticles came from microfluidic synthesis experiments. These experiments supplied particle populations of high homogeneity and high yield [16-18], which supported a rational interpretation of underlying mechanisms. As well as composed spherical metal nanoparticles [19], metal rods, cubes, polyeders [20] as well as flat nanotriangles had been prepared to a high quality using microfluidic techniques [21]. The control of electrical surface charges also allowed the preparation of different types of polymer/polymer and polymer/metal composite particles [22]. Some examples are shown in Figure 1. 


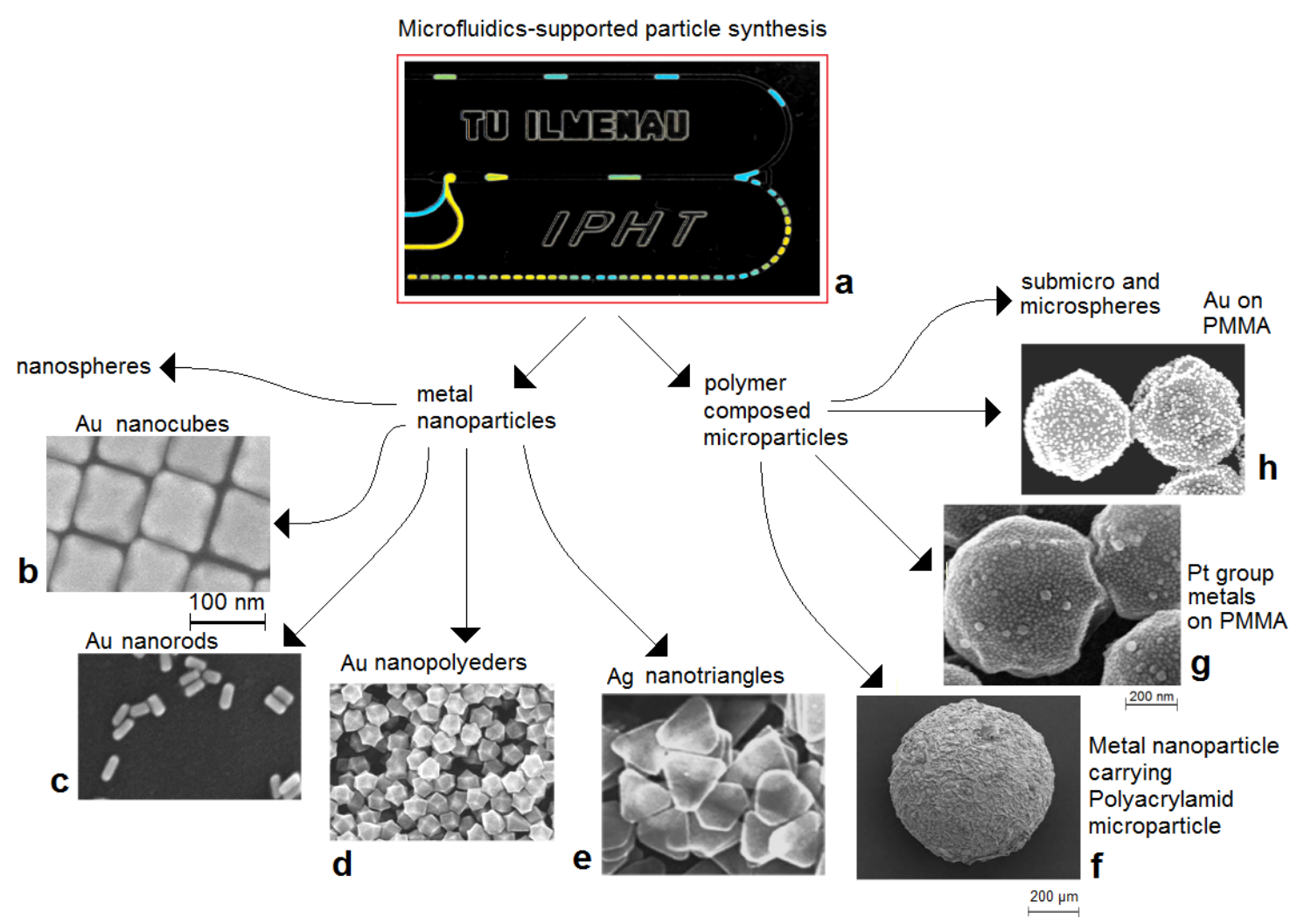

Figure 1. Examples of metal nano and polymer composite particles synthesized by use of microfluidic preparation steps: (a) example of a chip device for generation of microfluid segment sequences for systematic variation of reactant concentration ratios in micro flow syntheses [23], (b) gold nanocubes [20], (c) gold nanorods, (d) gold polyeders [20], (e) flat triangular silver nanoprisms [21], (f) polyacrylamid submillimeter composite particle [24], (g) submicrometer composite particle with two incorporated types of metal nanoparticles [25], (f) submicrometer composite particle with gold nanoparticles [24].

Since the days of the fundamental explaining of the nature of colored metal colloid solutions by Michael Faraday it has been clear that electricity plays a fundamental role in the formation and behavior of colloids [26]. In the following, how the conventional concept of mixed electrodes can contribute to a better understanding of the mechanisms of metal nanoparticle syntheses in liquid phase will be discussed.

\section{What Is Nucleation?}

In the classical La Mer model, nucleation is described as the primary aggregation of atoms or molecules of a supersaturated solution into small solids. The size of primary-formed nuclei is determined by the degree of supersaturation, the critical nucleation concentration, and the density of nuclei if this primary aggregation is regarded as a uniform process. The remaining supersaturation below the threshold of critical nucleation concentration is responsible for further growth of primary-formed particles.

While the Lamer model seems to be valid in several cases $[27,28]$, it is unable to explain all observations. Probably, the model is not applicable for the formation of all colloidal metal nanoparticles by chemical reduction of metal ions or complex compounds in liquid phase. It is safe to assume that the first metal-metal bonds are formed between ligand-bound metal atoms during the nucleation process. It seems to be less probable that a higher number of naked reduced metal atoms is firstly formed and distributed in the liquid phase and aggregated to small metal solids after achieving a critical density. 
Alternatively, a stepwise growth from the reduction of the first metal ion over the chemical formation and attachment of the second metal atom and a first stable cluster, which is successively growing by ongoing reductive metal deposition from the molecular-dispersive state of the chemical precursor, should be assumed.

In principle, the first direct binding between two metal atoms can be regarded as the ultimate step of nucleation in this case. It is proposed here to use the term "nucleation" in such a stepwise growth mechanism for the stage of particle formation in which a stable metal cluster is formed and chemical surface reaction can be connected with an exchange or transfer of electrons between different atoms of this cluster.

Based on this point of view, three different growth mechanisms or combinations of them can take place:

1. Particles are forming a regular primary crystal as the thermodynamically preferred state of minimum energy. Further stepwise growth results in the formation of colloidal solutions of regular crystals.

2. Particles are forming regular primary crystals as the thermodynamically preferred state of minimum energy, but the colloidal solution of primary-formed crystals is not stable. The primary-formed clusters aggregate in a very early stage, but they fuse together crystallographically regularly, resulting in larger regular crystals which are becoming stable and are forming a colloidal solution of larger regular crystals.

3. Particles are forming regular primary crystals as the thermodynamically preferred state of minimum energy, but the colloidal solution of primary-formed crystals is not stable, and aggregation occurs. However, this aggregation is non-regular, resulting in polynuclear particles with a random orientation of components. The aggregation and further deposition of metal atoms leads to a nearly spherical shape of metal nanoparticles [8,9] consisting of small crystal domains with different orientations ("Polte Mechanism").

\section{Colloidal Nanoparticles as "Open-circuit Mixed-Electrode Systems"}

The chemical reaction of metal ions or complex compounds of metal ions to metal atom is always a redox reaction. Frequently, the formation rates of seeds from a homogeneous reaction mixture are low or the nucleation is suppressed by a strong kinetic control. At a metal surface and even after the formation of nuclei, the redox process is enforced by the separation of metal ion reduction and oxidation of reducing agent due to an electron transfer via the preformed metal. The result is a strong autocatalytic activity of the metal taking place as it was observed in the chemical formation of $\mathrm{Pd}$ nanocubes [29].

In general, the redox reaction of the particle formation can be formulated for the simplest metal cluster formation in the following way by assuming a complex precursor involving $\mathrm{n}$ non-charged Ligands $L$ and a metal atom (M) with $m$ positive charges by a reducing agent (RA):

$$
2\left[\mathrm{ML}_{\mathrm{n}}\right]^{\mathrm{m}+}+(\mathrm{RA})_{\mathrm{red}} \rightarrow\left[\mathrm{M}_{2} \mathrm{~L}_{\mathrm{x}}\right]+(2 \mathrm{n}-\mathrm{x}) \mathrm{L}+(\mathrm{RA})_{\mathrm{ox}}{ }^{2 \mathrm{~m}+}(2 \mathrm{n} \geq \mathrm{x}),
$$

The essential step of this reaction is an electron transfer from the reducing agent in the reduced state $(R A)_{\text {red }}$ to the metal precursor species $\left[\mathrm{ML}_{n}\right]^{\mathrm{m}+}$ under formation of the primary metal cluster $\left[\mathrm{M}_{2} \mathrm{~L}_{\mathrm{x}}\right]$ and the oxidized reduced agent $(\mathrm{RA})_{\mathrm{ox}}{ }^{2 \mathrm{~m}+}$. The cluster formation is accompanied by a deliberation of ligands $L$ if a complex species is the precursor.

The above-described reaction can be subdivided into two partial reactions, the reduction reaction:

$$
2\left[M L_{n}\right]^{m+}+m e^{-} \rightarrow\left[M_{2} L_{x}\right]+(2 n-x) L(2 n \geq x),
$$

and the oxidation reaction:

$$
(\mathrm{RA})_{\text {red }} \rightarrow \mathrm{m} \mathrm{e}^{-}+(\mathrm{RA})_{\mathrm{ox}}{ }^{\mathrm{m}+},
$$


Both these partial reactions take place in further steps of reactive metal deposition on the growing particles. In case of the existing of a cluster or particle, it can be regarded as an electrode. Then both partial reactions (2) and (3) can proceed in the form of electrode reactions: process (2) represents the cathodic partial process, reaction (3) the anodic partial process. The particle then has the function of a mixed electrode of two electrode processes. It forms an open-circuit system because all charge exchanges are caused by electrochemical processes and are not related to any outside wiring. The mixed-potential approach is consistent with well-known effects from macroscopic metal deposition processes by electroless plating. It can also be applied for interpretation of the growth of different types of nanoparticles during the metal deposition on a solid surface as in case of silver nanoplatelets [30].

The open-circuit potential $U_{0}$ of the particle mixed electrode is formed by the balance of the charge exchange the anodic and the cathodic current $\mathrm{I}_{+}$and $\mathrm{I}_{-}$:

$$
\mathrm{I}_{+}+\mathrm{I}_{-}=0
$$

Assuming an exponential dependence of partial currents from electrode potential U (Tafel equation, which can be regarded as a special form of Nernst equation):

$$
\mathrm{I}_{+}=\mathrm{I}_{+0} \times \mathrm{e}^{\mathrm{a} \times(\mathrm{U}-\mathrm{Ua} 0)},
$$

and:

$$
\mathrm{I}_{-}=-\mathrm{I}_{-0} \times \mathrm{e}^{-\mathrm{b} \times(\mathrm{U}-\mathrm{Ub} 0)},
$$

(with $\mathrm{a}=\mathrm{z}_{+} \times \mathrm{F} /(\mathrm{R} \times \mathrm{T}), \mathrm{b}=\mathrm{z}_{-} \times \mathrm{F} /(\mathrm{R} \times \mathrm{T}), \mathrm{F}=$ Faraday constant, $\mathrm{R} \times \mathrm{T}=$ thermal energy, $\mathrm{z}_{-}, \mathrm{z}_{+}=$ electrochemical valence, and $\mathrm{U}_{\mathrm{a} 0}$ and $\mathrm{U}_{\mathrm{b} 0}$ standard potentials of the anodic and the cathodic process) there is obtained for $\mathrm{U}_{0}$ :

$$
\mathrm{I}_{+0} \times \mathrm{e}^{\mathrm{a} \times\left(\mathrm{U}-\mathrm{U}_{\mathrm{a} 0}\right)}=-\mathrm{I}_{-0} \times \mathrm{e}^{-\mathrm{b} \times(\mathrm{U} 0-\mathrm{Ub} 0)},
$$

or

$$
\mathrm{U}_{0}=\left\{\left(\mathrm{U}_{\mathrm{b} 0}+\mathrm{U}_{\mathrm{a} 0}\right)+\ln \left(\mathrm{I}_{+0} \div-\mathrm{I}_{-0}\right)\right\} \div(\mathrm{b}+\mathrm{a})
$$

In summary, it can be said that standard potentials and intensities of anodic and cathodic partial processes determine the resulting open-circuit potential of a growing colloidal metal particle: an increase of cathodic partial process intensity (metal ion reduction) means an increase of electron consumption and, therefore, an increase of electrode potential, for example caused by an increase of metal ion concentration. An increase of anodic partial process intensity (oxidation of reducing agent) means an increase of electron supply and, therefore, a decrease of electrode potential, for example caused by an increasing concentration of reducing agent [31].

The electrode concept allows a rough estimation of the number of electrical charges $\mathrm{N}$ of a particle by the particle surface area $\mathrm{A}$, the electrical field constant $\varepsilon$, the thickness of electrochemical double layer $\mathrm{d}$, the elementary charge $\mathrm{q}_{\mathrm{e}}$ and the particle potential:

$$
\mathrm{N}=\mathrm{Q} / \mathrm{q}_{\mathrm{e}}=\varepsilon / \mathrm{q}_{\mathrm{e}} \times \mathrm{A} / \mathrm{d} \times \mathrm{U}
$$

Assuming a spherical particle of $2 \mathrm{~nm}$ diameter a surface area of about $12 \mathrm{~nm}^{2}$, a double layer thickness of about $0.5 \mathrm{~nm}$ and a potential of $40 \mathrm{mV}$ results and under approximation by the field constant of water result a charging by about 4 electrons only:

$$
\mathrm{N}_{(2 \mathrm{~nm}, 50 \mathrm{mV})} \approx 4
$$

This number is enhanced to about 42 in case of a particle of $20 \mathrm{~nm}$ diameter. Despite the fact that such particles contain thousands of atoms, their electrical behavior is significantly affected by a comparatively low number of excess electrons. 


\section{Spatial Decoupling of Electrochemical Partial Processes}

The mixed-electrode concept includes two important aspects of electrochemical behavior of the nanoparticles: First, the formation of the open-circuit potential corresponds to a certain netto charge of the particle. This netto charge is determined by the anodic and cathodic electrode processes on the one hand and by the adsorption of charged ligands or other ions on the other hand. Second, both partial processes are always connected by the electron conductivity of the particle but can proceed at different surface sites. This means that the anodic and the cathodic partial process are spatially de-coupled. The partial processes form a common electrochemical potential. It is determined by the intensity of the both antagonistic partial processes. All cathodic partial current is compensated completely by the corresponding anodic processes. This situation can be illustrated by the current/voltage function (Figure 2a). In case of a partial local decoupling; there arises a certain asymmetry between anodic and cathodic activity at different surface areas of the particle if the different electrode activities are compensated by an electrical current over the particle (Figure $2 b, c$ ).

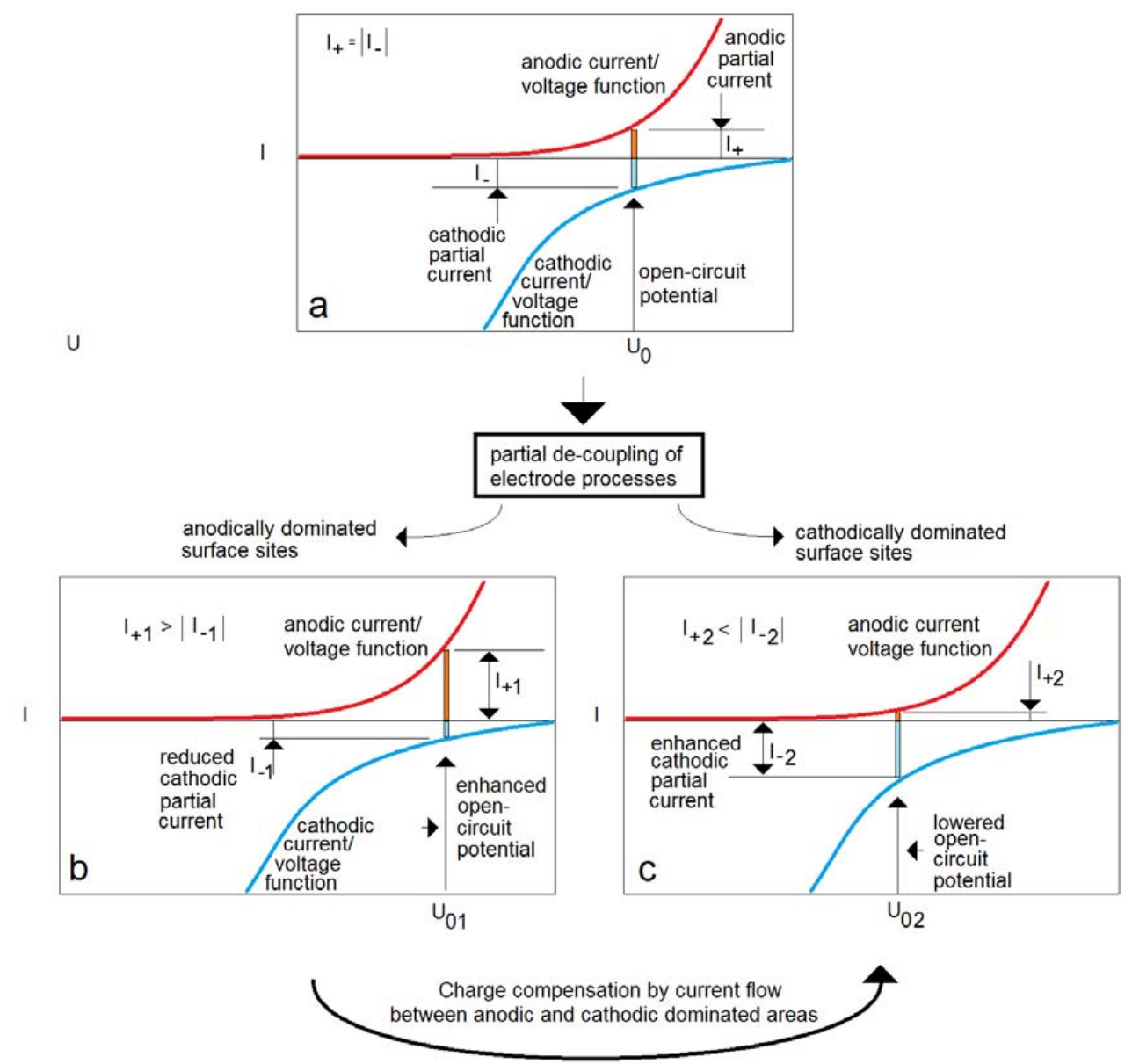

Figure 2. Electrochemical model of a growing or corroding metal nanoparticle in colloidal solution; Current/voltage characteristics of open-circuit electrochemical systems: (a) complete complementary compensation of anodic and cathodic partial processes at the particle potential $U_{0},(\mathbf{b})$ dominance of anodic partial processes on a surface part of a metal nanoparticle in case of a local current to cathodically dominated surface regions, (c) dominance of cathodic partial processes on a surface part of a metal nanoparticle in case of a local current to anodically dominated surface regions.

With respect to different situations of surface atoms of a metal nanoparticle and to different local adsorption of ligands, it is very probable that there exist special but different sites for the anodic and the cathodic partial process on the particle surface. It is safe to assume that the sites of the cathodic 
partial process (metal deposition by reduction of metal ions) show a much stronger spatial fluctuation than the sites of anodic partial process. This assumption is deviated from the fact that the cathodic process itself modifies permanently the surface state and surface shape due to the deposition of the metal ions. This process is modifying the local surface structure permanently. The geometric and thermodynamic conditions of the attachment and reduction of metal ions on the particle surface are permanently changing due to the particle growth. A typical consequence could be a mechanism of cathodic metal deposition determined by stochastic adsorption of metal ions followed by formation of atomic steps and growing atomic steps by filling of atomic layers in local crystalline order.

In contrast, the anodic partial process of oxidation of reducing agent can be repeated on a thermodynamically preferred place if no metal ion deposition or other adsorption processes occupies these places by random fluctuations. It must be expected that places which are thermodynamically slightly preferred for the adsorption of molecules of the reducing agent, or which are marked by a slightly reduced local electron density, present active sites for the fast ongoing anodic partial process.

\section{Self-Polarization of Non-Spherical Metal Nanoparticles}

The structure of nanocrystals is mainly determined by the crystallographic arrangement of involved atoms to form thermodynamically stable state and the resulting intrinsic anisotropy. In addition, the shape of the forming nanostructures can be affected by adsorbing surfactants or other ligands and can be influenced by the spontaneous formation of defects. In case of an electrically conductive material as in the case of metal nanoparticles, electrostatic effects are an additional important factor for the formation of certain nanoparticle geometries.

The frequency of elementary charge transfer steps at the individual surface points at a nanoparticle surface is dependent on the local electron density and the probability of adsorption of involved molecules or ions. This local electron surface density is determined by the atomic structure of metal surface and can be influenced by intermediate adsorbing ligands. Therefore, the character and reactivity of each place is determined by the immediate atomic environment, on the one hand.

On the other hand, the reactivity of local sites is influenced by the excess charge of the particle, which means by its electrochemical potential. A more positive electrochemical potential of particle enforces anodic processes and reduces the probability of cathodic processes. A reduction of electrochemical potential enhances the probability of cathodic processes and reduces the frequency of anodic.

In contrast to bulk metal electrodes, a metal nanoparticle must be regarded as a polarizable system. Thus, the distribution of excess charge is dependent on the geometry of the particle. In the case of a spherical particle, a homogeneous distribution of excess charges at the particle surface must be expected. It results from the charge repulsion and the minimization of electrostatic surface energy.

The charge distribution is completely changed in the case of charged non-spherical metal nanoparticles. The repulsion of electrons (in case of negatively charged particles) or holes (in case of positive excess charge) leads to local differences in charge density. The electrostatic repulsion pushes the excess charge of rod-shaped metal nanoparticles towards the poles. The excess charge of circular discs is concentrated on the periphery of the particles. The electrical excess charges are pushed towards the corners in triangular, cube-like or other prismatic particles.

This effect of self-polarization leads to an asymmetry in the local probability of anodic and cathodic partial processes: in the case of a positive excess charge, anodic partial processes are mainly to be expected at the particle periphery, at edges, poles, and corners. In the case of a negative excess charge, these sites are the preferential places for the cathodic partial processes. In both cases, charged non-spherical metal nanoparticles are marked by a complementary local distribution of the reaction rates of potential forming anodic and cathodic partial processes.

The wet chemical synthesis of metal nanoparticles by reduction of metal salt or complex compounds is frequently performed with an excess of reducing agent. Typically, the colloidal solution of product particles is marked by a negative Zeta potential reflecting a negative charge of the formed 
metal nanoparticles. In the case of non-spherical nanoparticles, the deposition of metal atoms by reduction of the precursor species takes place preferentially at the corners and edges. The result is the non-spherical character of particles being promoted: rods become longer and tend to form higher aspect ratios, for example. Triangular nanoprisms tend to evolve into flat extended shapes. In contrast, a preferential deposition of metal can take place at the mantle of nanorods in the case of negatively charged metal precursor species or by a slightly positive particle potential (Figure 3). A detailed study on the effect of concentration variation of metal precursors (principle component in the cathodic partial process) and of reducing agent (principle component in the anodic partial process) on the axial and the radial growth of nanorods and on the resulting aspect ratio could help to verify the applicability of the mixed-potential approach. Concentration-dependent measurements on Zeta potentials of obtained colloidal solutions can further support such an interpretation. In general, electrical charges and self-polarization of charged non-spherical nanoparticles are key issues for the local deposition (or corrosion) rates of metal nanoparticles and can control the finally formed particle geometry. The concept of self-polarization can be used for the interpretation of experimental data on the growth of silver nanotriangles with sharp corners by growth of silver seeds in the presence of the polyanionic macromolecule Polystyrene sulfonic acid [22]. The negative functional groups of the polyanionic molecules push the negative charges into the corners of the triangles and cause an enhanced attraction of silver ions by the particle corners and the preferential deposition of silver in the corner region. Obviously, the edges are filled by silver atoms after the metal deposition at the corners. The self-polarization concept is also supported by experiments on the substitute on of PSS by thiocyanate, which causes a spontaneous transformation of nanotriangles in to compact nanoparticles [32]. This $\mathrm{SCN}^{-}$-dependent spontaneous conversion is reflected by optical spectra, SEM images and Zeta potential measurements. It could be concluded that the electrical excess charge is mainly responsible for the stabilization of the flat silver triangles by electrostatic forces.

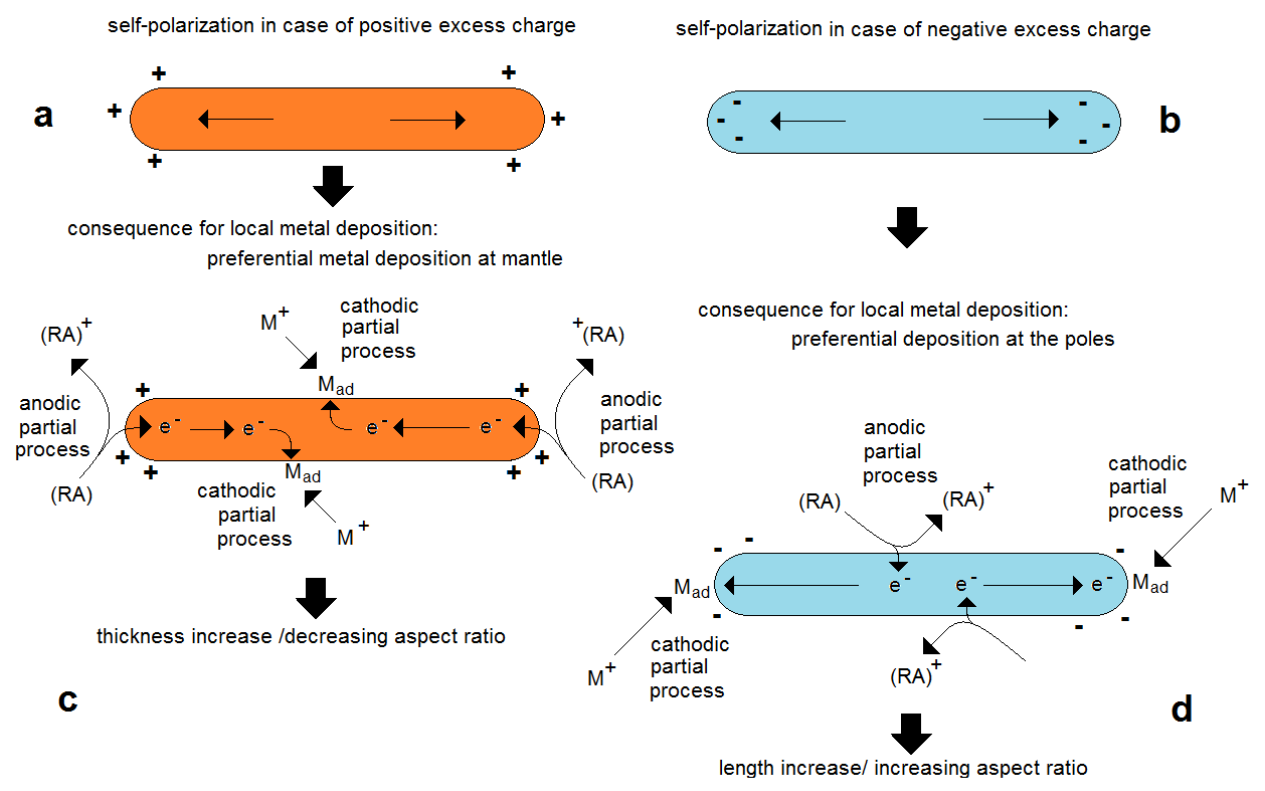

Figure 3. Effect of self-polarization on the evolution of particle shape; example of a growing metal nanorod. positive charges are pushed into direction of the poles of the metal nanorod in case of a positive excess charge (a) causing the preferential oxidation of reducing agent molecules (RA) at the particle poles (c); negative charges are pushed into direction of the poles of the metal nanorod in case of a negative excess charge $(\mathbf{b})$ causing the preferential reduction of positively charged metal ions causing the deposition of metal (0) at the particle poles (d). 
At the positive potential, in contrast, corners and edges are the sites where corrosion takes place preferentially. Corroding flat triangles lose their corners and are forming circular discs in the case of corrosion. Cubes react by the formation of rounded shapes, too.

The electrical charging and self-polarization of flat nanoparticles such as triangular prisms and disks is obviously responsible for the stabilization of particles with high aspect ratios [32]. Such particles can be generated and stabilized by the influence of polyanionic macromolecules [33]. A ligand-induced removal of these molecules leads to the collapse of the flat particles into compact shape [32].

\section{Potential-Controlled Particle Shapes}

The dense packaging of metal atoms is the reason for the formation of cubic and hexagonal crystals of metals. This effect is responsible for the crystalline structure of bulk metal material-single-crystalline as well as poly-crystalline-and for the primary structure of metal nanoparticles. It causes the formation of nanocrystals with the shape of cubes, hexagons, and triangles, if no spontaneous aggregation of nanocrystals takes place. Despite crystallographic reasons, a spontaneous symmetry break can be crucial for the formation of non-spherical particles, in particular non-compact particles with high aspect ratios. A spontaneous symmetry break can be due to the stochastic character of nanoparticle formation, small fluctuations, and can be related to lattice misfits or other single defects in the particle growth [34].

High dilution of colloidal solution-low particle concentration-and high particle charge are the main factors for prevent particle aggregation [35]. Vice versa, moderate electrical charging can be used for controlling the growth of particle assemblies [36].

Aggregation of nanocrystals can take place if the thermal mobility of particles is high enough to overcome the electrostatic repulsion. Therefore, aggregates and larger coagulates are formed if the electrical charge of particles is small. The probability of particle/particle contacts and aggregation is high in the early phase of particle growth. This is due to the fact that small particles are mobile one the one hand, and the electrochemical potential tends to be more positive in the start phase of particle growth if the concentration of the metal precursor in the solution is still comparatively high. In some cases, the electrochemical potential or particles might be transiently going from positive charging to negative charging during the lowering of metal ion concentration, which causes a zero-transition for the particle charge with high aggregation tendency.

The aggregates are much slower in their thermal motion than the primarily formed small nanocrystals. This can lead to a re-stabilization of the colloidal state. During the growth process of particles, this re-stabilization is additionally supported by an increase of negative particle charge caused by the lowering of electrochemical potential due to the consumption of the potential-enhancing metal precursor. A certain final particle growth by deposition of metal atoms after the particle aggregation and colloidal re-stabilization results in nearly spherical shapes of nanoparticles. These particles have a polynuclear morphology, typically (Figure 4). This morphology is caused by the random orientation of crystal planes during the aggregation of small nanocrystals. Highly resolved transmission electron images confirm the presence of small crystallites with different orientations inside spherical metal nanoparticles. The whole mechanism of formation of spherical metal nanoparticles was proved by Polte et al. for the chemical flow synthesis of gold nanoparticles by application of in situ X-ray characterization [9].

The decision between the formation of colloids of nanocrystals or colloids of polynuclear spheres is dependent on the temporal evolution of particle growth and electrochemical particle potential. The latter is influenced by the intensities of anodic and cathodic partial processes, but in addition determined by the interaction of particles with ligands. The charge of nanoparticles can strongly dependent on ligands if they are charged. Ligands with a high chemical affinity to the metal surface and ligands carrying higher numbers of electrical charges are efficient for the control of particle charge and, therefore, for the direction of the evolution of particle shape by self-polarization and aggregation. 


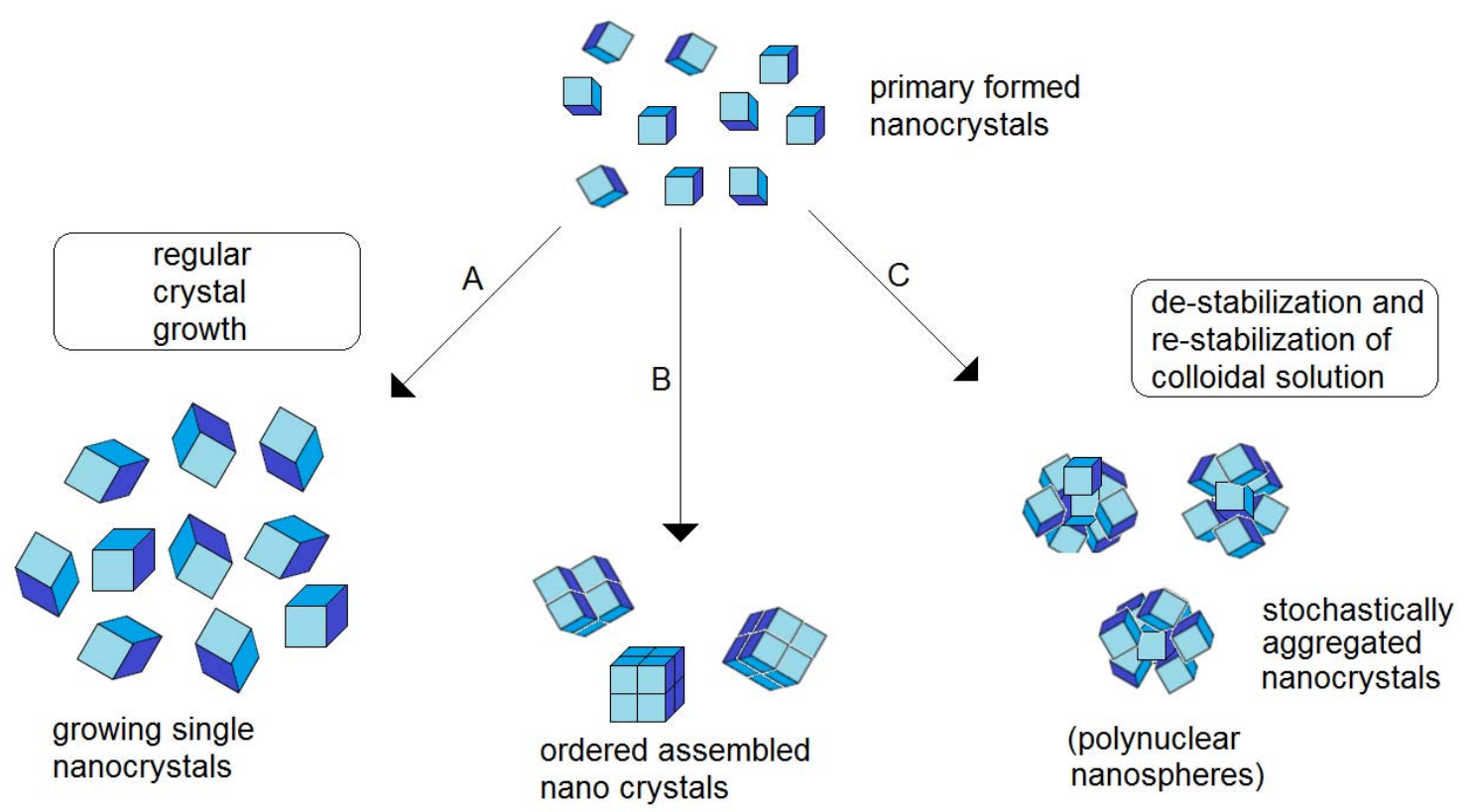

Figure 4. Potential-dependent behavior of forming metal nanoparticles in colloidal solution (schematically): Single crystals are obtained if stronger repulsive forces and/or solvation inhibit the interaction and agglomeration of particles (A), more or less regular particle assemblies can be obtained in case of an aggregation with a partial surface mobility of assembling components (B), stochastic aggregation without mobility causes the formation of irregular composed particles which might be self-stabilized above a critical particle size (C). Ongoing metal deposition leads to the formation of nearly spherical polynuclear nanoparticles [8].

The electrochemical conditions decide first about the formation of compact or high-aspect-ratio crystals: in the case of a positive particle potential, the deposition of metal atoms from metal ions on corners, poles and edges is suppressed. As a result, compact nanocrystals as cubes or polyeders are formed.

This rule might only be violated if the deposition of metal is transport-controlled and the metal precursor is negatively charged. This is the case if negatively charged complex ions are used; for example, if tetrachloroaurate is applied for the synthesis of gold nanoparticles. This can lead to the growth of rod-like nanoparticles in case of the application of a positively charged ligand with high surface affinity; for example tetralkylammonium ions as cetyltriethylammoniombromide. In the case of negative excess charge, the metal deposition from metal ions has its highest rate at corners, poles, and edges. As a result, flat high-aspect-ratio crystals such as flat hexagons or triangles are formed.

\section{Conclusions}

The concept of an open-circuit mixed potential is applicable for the interpretation of growth, properties, and behavior of metal nanoparticles in colloidal solutions. It allows understanding of the formation of the electrochemical particle potential and interpretation of the development of non-spherical particles by repulsion of electrical excess charges. The resulting self-polarization is accompanied by a partial local decoupling of anodic and cathodic partial processes and explains the formation of sharp edges and corners during the particle growth. The concepts of open-circuit mixed-potential formation and self-polarization is also applicable for the explanation of the rounding of corners in case of corrosion processes on non-spherical metal particles. Finally, the sight on the electrical soul of the metal nanoparticles in colloidal solutions allows understanding of the high variety of particle shapes and morphologies by the interaction of electrochemical processes, particle/ligand reactions, and particle/particle interactions. 
Author Contributions: Conceptualization, J.M.K. and A.K.; Experiments, A.K.; Writing-Original Draft Preparation, J.M.K.

Funding: This research was funded by [Deutsche Forschungsgemeinschaft] grant number [KO 1403-39/3]

Acknowledgments: We thank J. Polte (Berlin) for discussion on nucleation and in situ aggregation of nanoparticles. We are very grateful for the collaboration and support of Nikunjkumar Visaveliya, Xiang Li, Lars Hafermann, Danja Kuhfuss (Ilmenau), Christophe Serra (Strasbourg), Wolfgang Fritzsche, Thomas Henkel, Mark Kielpinski and Andrea Csaki (Jena). The financial support of "Deutsche Forschungsgemeinschaft" (KO 1403-39/3) for investigations on non-spherical and composed metal nanoparticles is gratefully acknowledged.

Conflicts of Interest: The authors declare no conflict of interest.

\section{References}

1. Henglein, A. Small-particle research: physicochemical properties of extremely small colloidal metal and semiconductor particles. Chem. Rev. 1989, 89, 1861-1873. [CrossRef]

2. Csaki, A.; Kaplanek, P.; Möller, R.; Fritzsche, W. The optical detection of individual DNA-conjugated gold nanoparticle labels after metal enhancement. Nanotechnology 2003, 14, 1262-1268. [CrossRef] [PubMed]

3. Mpourmpakis, G.; Vlachos, D.G. Insights into early stages of metal nanoparticle formation via first-principle calculations: The role of citrate and water. Langmuir 2008, 24, 7465-7473. [CrossRef] [PubMed]

4. Lofton, C.; Sigmund, W. Mechanism controlling crystal habits of gold and silver colloids. Adv. Funct. Mater. 2005, 15, 1197-1208. [CrossRef]

5. Hubert, F.; Testard, F.; Rizza, G.; Spalla, O. Nanorods versus nanospheres: A bifurcation mechanism revealed by principal component TEM analysis. Langmuir 2010, 26, 6887-6891. [CrossRef] [PubMed]

6. Fu, Y.Z.; Du, Y.K.; Yang, P.; Li, J.R.; Jiang, L. Shape-controlled synthesis of highly monodisperse and small size gold nanoparticles. Sci. China B 2007, 50, 494-500. [CrossRef]

7. Rai, A.; Singh, A.; Ahmad, A.; Sastry, M. Role of halide ions and temperature on the morphology of biologically synthesized gold nanotriangles. Langmuir 2005, 22, 736-741. [CrossRef] [PubMed]

8. Polte, J.; Kraehnert, R.; Radtke, M.; Reinholza, U.; Riesemeier, H. New insights of the nucleation and growth process of gold nanoparticles via in situ coupling of SAXS and XANES. J. Phys Conf. Ser. 2010, 247, 012051. [CrossRef]

9. Polte, J.; Ahner, T.T.; Delissen, F.; Sokolov, S.; Emmerling, F.; Thünemann, A.F.; Kraehnert, R. Mechanism of gold nanoparticle formation in the classical citrate synthesis method derived from coupled in situ XANES and SAXS evaluation. J. Am. Chem. Soc. 2010, 132, 1296-1301. [CrossRef] [PubMed]

10. Köhler, J.M.; Romanus, H.; Hübner, U.; Wagner, J. Formation of star-like and core-shell nanoparticles during two- and three-step preparation in batch and in microfluidic systems. J. Nanomater. 2007, 1, 2. [CrossRef]

11. Zhang, Q.; Li, N.; Goebl, J.; Lu, Z.D.; Yin, Y.D. A systematic study of the synthesis of silver nanoplates: is citrate a "magic" reagent? J. Am. Chem. Soc. 2011, 46, 18931-18939. [CrossRef] [PubMed]

12. Dougherty, G.M.; Klint, A.R.; Tok, J.B.H.; Pannu, S.S.; Chuang, F.Y.S.; Sha, M.Y.; Chakarova, G.; Penn, S.G. The zeta potential of surface-functionalized metallic nanorod particles in aqueous solution. Electrophoresis 2008, 29, 1131-1139. [CrossRef] [PubMed]

13. Sun, Y.; Xia, Y. Shape-controlled synthesis of gold and silver nanoparticles. Science 2002, 298, $2176-2179$. [CrossRef] [PubMed]

14. Murphy, C.J.; San, T.K.; Gole, A.M.; Orendorff, C.J.; Gao, J.X.; Gou, L.F.; Hunyadi, S.E.; Li, T. Anisotropic metal nanoparticles: synthesis, assembly, and optical applications. J. Phys. Chem. B 2005, 109, 13857-13870. [CrossRef] [PubMed]

15. Smith, D.K.; Korgel, B.A. The importance of the CTAB surfactant on the colloidal seed-mediated synthesis of gold nanorods. Langmuir 2008, 24, 644-649. [CrossRef] [PubMed]

16. Shestopalov, I.; Tice, J.D.; Ismagilov, R.F. Multistep synthesis of nanoparticles performed on millisecond time scale in a microfluidic droplet-based system. Lab. Chip 2004, 4, 316-321. [CrossRef] [PubMed]

17. Wagner, J.; Köhler, J.M. Continuous synthesis of gold nanoparticles in a microreactor. Nano Lett. 2005, 5, 685-691. [CrossRef] [PubMed]

18. Duraiswamy, S.; Khan, S.A. Droplet-based microfluidic synthesis of anisotropic metal nanocrystals. Small 2009, 5, 2828-2834. [CrossRef] [PubMed] 
19. Knauer, A.; Thete, A.; Li, S.; Romanus, H.; Csáki, A.; Fritzsche, W.; Köhler, J.M. Au/Ag/Au double shell nanoparticles with narrow size distribution obtained by continuous micro segmented flow synthesis. Chem. Eng. J. 2011, 166, 1164-1169. [CrossRef]

20. Thiele, M.; Soh, J.Z.E.; Knauer, A.; Malsch, D.; Stranik, O.; Müller, R.; Csáki, A.; Henkel, T.; Köhler, J.M.; Fritzsche, W. Gold nanocubes-Direct comparison of synthesis approaches reveals the need for a microfluidic synthesis setup for high reproducibility. Chem. Eng. J. 2016, 38, 432-440. [CrossRef]

21. Knauer, A.; Csaki, A.; Möller, F.; Huhn, C.; Fritzsche, W.; Köhler, J.M. Microsegmented flow-through synthesis of silver nanoprisms with exact tunable optical properties. J. Phys. Chem. C 2012, 116, 9251-9258. [CrossRef]

22. Köhler, J.M.; Visaveliya, N.; Knauer, A. Controlling formation and assembling of nanoparticles by control of electrical charging, polarization, and electrochemical potential. Nanotechnol. Rev. 2012, 116, 553-568. [CrossRef]

23. Henkel, T.; Bermig, T.; Kielpinski, M.; Grodrian, A.; Metze, J.; Köhler, J.M. Chip modules for generation and manipulation of fluid segments for micro serial flow. Chem. Eng. J. 2004, 101, 439-445. [CrossRef]

24. Visaveliya, N.; Knauer, A.; Wei, Y.; Serra, C.A.; Köhler, J.M. Microflow-assisted assembling of multiscale polymer particles by controlling surface properties and interactions. Eur. Polym. J. 2016, 80, $256-267$. [CrossRef]

25. Li, X.; Visaveliya, N.; Hafermann, L.; Gross, G.A.; Knauer, A.; Köhler, J.M. Hierarchically structures particles for micro flow catalysis. Chem. Eng. J. 2017, 326, 1058-1065. [CrossRef]

26. Edward, P.P.; Thomas, J.M. Gold in a metallic state-From Faraday to present-day nanoscience. Angew. Chem. Int. Ed. 2007, 46, 5480-5486. [CrossRef] [PubMed]

27. Kim, B.H.; Yang, J.; Lee, D.; Choi, B.K.; Hyeon, T.; Park, J. Liquid-phase transmission electron microscopy for studying colloidal inorganic nanoparticles. Adv. Mater. 2018, 30, 1703316. [CrossRef] [PubMed]

28. Chen, Y.-C.; Chen, J.-Y.; Wu, W.W. In situ observation of Au nanostructure evolution in liquid cell TEM. J. Phys. Chem. C 2017, 121, 26069-26075. [CrossRef]

29. Yang, T.-H.; Peng, H.-C.; Zhou, S.; Lee, C.T.; Bao, S.X.; Lee, Y.H.; Wu, J.M.; Xia, Y. Toward a quantitative understanding of the reduction pathways of salt precursor in the synthesis of metal nanocrystals. Nano Lett. 2017, 17, 334-340. [CrossRef] [PubMed]

30. Muench, F.; Vaskevich, A.; Popovitz-Biro, R.; Bendikov, T.; Feldman, Y.; Rubinstein, I. Expanding the boundaries of metal deposition: High aspect ratio silver platelets created by merging nanobelts. Electrochim. Act. 2018, 264, 233-243. [CrossRef]

31. Köhler, J.M.; Knauer, A. The electrical soul of metal nanoparticles-What experiments with microfluidically prepared colloids tell us. Adv. Nano-Bio-Mater. Devices 2017, 1, 1-17.

32. Knauer, A.; Visaveliya, N.; Köhler, J.M. Spontaneous transformation of polyelectrolyte-stabilized silver nanoprisms by interaction with thiocyanate. J. Colloid. Interf. Sci. 2013, 394, 78-84. [CrossRef] [PubMed]

33. Aherne, D.; Ledwith, D.M.; Gara, M.; Kelly, J.M. Optical properties and growth aspects of silver nanoprisms produced by a highly reproducible and rapid synthesis at room temperature. Adv. Funct. Mater. 2008, 18, 2005-2016. [CrossRef]

34. Gilroy, K.D.; Peng, H.-C.; Yang, X.; Ruditsky, A.; Xia, Y. Symmetry breaking during nanocrystal growth. Chem. Comm. 2017, 53, 4530. [CrossRef] [PubMed]

35. Meyer, M.; Le Ru, E.C.; Etchegoin, P.G. Self-limiting aggregation leads to long-lived metastable clusters in colloidal solution. J. Phys. Chem. B 2006, 110, 6040-6047. [CrossRef] [PubMed]

36. Zhang, H.; Wang, D. Controlling the growth of charged-nanoparticle chains through interparticle electrostatic repulsion. Angew. Chem. Int. Ed. 2008, 47, 3984-3987. [CrossRef] [PubMed]

(C) 2018 by the authors. Licensee MDPI, Basel, Switzerland. This article is an open access article distributed under the terms and conditions of the Creative Commons Attribution (CC BY) license (http://creativecommons.org/licenses/by/4.0/). 